\title{
Research and Implementation of the Automatic Detection System of Aggregate Particles Characteristic Parameters Based on VC
}

\author{
Zhaoyun Sun \\ Information Engineering School \\ Chang'an University \\ Xi'an, China \\ e-mail: zhaoyunsun@126.com
}

Wei Li

Information Engineering School

Chang'an University

Xi'an, China

\begin{abstract}
For the purpose of obtaining the characteristic parameters of the asphalt mixture aggregate particles, this paper put forward a viable method using two-dimensional image. The automatic detection system of asphalt mixture aggregate characteristic parameters is developed based on $\mathrm{VC}++$. A series of processing are done on the aggregate images collected, including image edge detection, image segmentation, morphological processing, region-labeling, contouring and so on .The region-labeling algorithm is improved ending with low complexity, high robustness. Database technology is used to deal with the storage, query of the results and several other operations. The whole system characterized with perfect function, user-friendly interface and stable performance, providing a practical and accurate method for the characteristic detection of asphalt mixture aggregate particles.
\end{abstract}

Keywords-aggregate particles detection; characteristic parameters; image segmentation; region-labeling; contour tracking; database.

\section{INTRODUCTION}

Nowadays, asphalt pavement is the main form of China's high-grade highways and people's requirements for the quality of roads are getting higher. Operation performance of asphalt mixture which is the major kind of road construction material is drawing more and more attention. Existing asphalt mixture aggregate particles detection methods are empirical, their inadequate theoretical foundation and outdated technology results in the complex process and low efficiency of detection which will inevitably lead to many mistakes, a serious waste of human and material resources. The characteristic detection method explore using digital image processing technology [1,2] has its theoretical significance and obvious economic benefits.

Based on the applied research of digital image technology in the aggregate particles image processing, authors developed a particle image processing system under Visual Studio 2008 and the SQLServer2000. Complete functions as follows: image preprocessing [3, 4], including graying and image enhancement $[5,6]$; image segmentation, including edge detection, binarization and morphological processing $[7,8]$; parameter extraction, including

\author{
Xueli Hao \\ Information Engineering School \\ Chang'an University \\ Xi'an, China \\ e-mail: 460302526@qq.com
}

\author{
Mengxia Yuan \\ Information Engineering School \\ Chang'an University \\ Xi'an, China
}

region-labeling and calculation of characteristic parameters; contour drawing, depicting the outline of the particles; database module, realize storage, query and other operations[9]. Region-labeling algorithm [10] was improved, so this system can obtain more accurate characteristic parameters of particles in the end.

\section{Design Of The AgGREgAte Particles IMAGE PROCESSING SYSTEM}

To analyze the asphalt mixture image and acquire characteristic parameters of asphalt mixture aggregate, the system was developed under Visual Studio 2008 environment based on OOP (object-oriented programming) design ideas. With its powerful function, easy operation, and various interfaces provided to all kinds of client-side development tools, SQL Server 2000 is used widely. So storage and management of data were achieved used SQL Server 2000, which is also conducive to in-depth data analysis and data mining.

The original collected image is generally in the form of 24-bit true color and it is converted to a gray-scale image as the first step. In the process of image acquisition and transmission, much noise were introduced to the image inevitably due to various uncontrollable factors, so in order to improve its visual effects and suppress those noise, image enhancement and image smoothing technique is necessary. Secondly, extract the edge of the aggregate particles. To obtain better results, image segmentation and binarization were done successively. To a certain extent, aggregate particles have adhesion among each other which can be effectively eliminated using morphological methods. Then achieve parameter extraction, expression as well as contour extraction by particle analysis. Finally, database is designed to accomplish storage, query and report printing of processing results.

\section{System PRocessing AND FunCtion Module}

\section{A. Software Process}

The main part of this system is realized based on VC single document in Visual Studio 2008. The system 
identifies the best way for aggregate image from available processing methods in the various stages of image processing, thus achieve feature extraction of aggregate particles. This paper introduces the software's operating procedure in detail from image inputting, pre-processing to post-processing. The overall process is shown in Fig. 1.

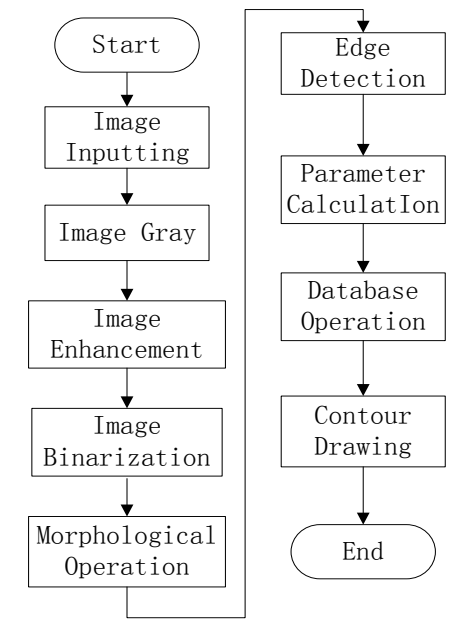

Figure 1. System process chart

\section{B. System Structure And Working Principle}

The system block diagram is shown in Fig. 2.

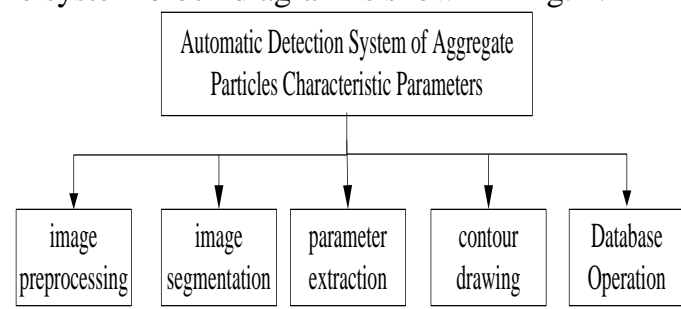

Figure 2. $\quad$ system block diagram

Images are inevitably affected by noise during the process of collecting and processing, so it is necessary to do preprocessing in order to minimize noise, improve image quality and accuracy of the asphalt mixture detection.

Considering the characteristics of asphalt mixture and requirements for reduce the amount of calculation, it is better to treat grayscale images as the analysis objects. Since the collected image is color image, it should first be converted to a grayscale image and do image enhancement. Then the system takes advantage of smoothing technology to minimize the impact of noise on the follow-up study. The image preprocessing module design is shown in Fig. 3. The preprocessed image corresponding to the one is shown in Fig. 4.
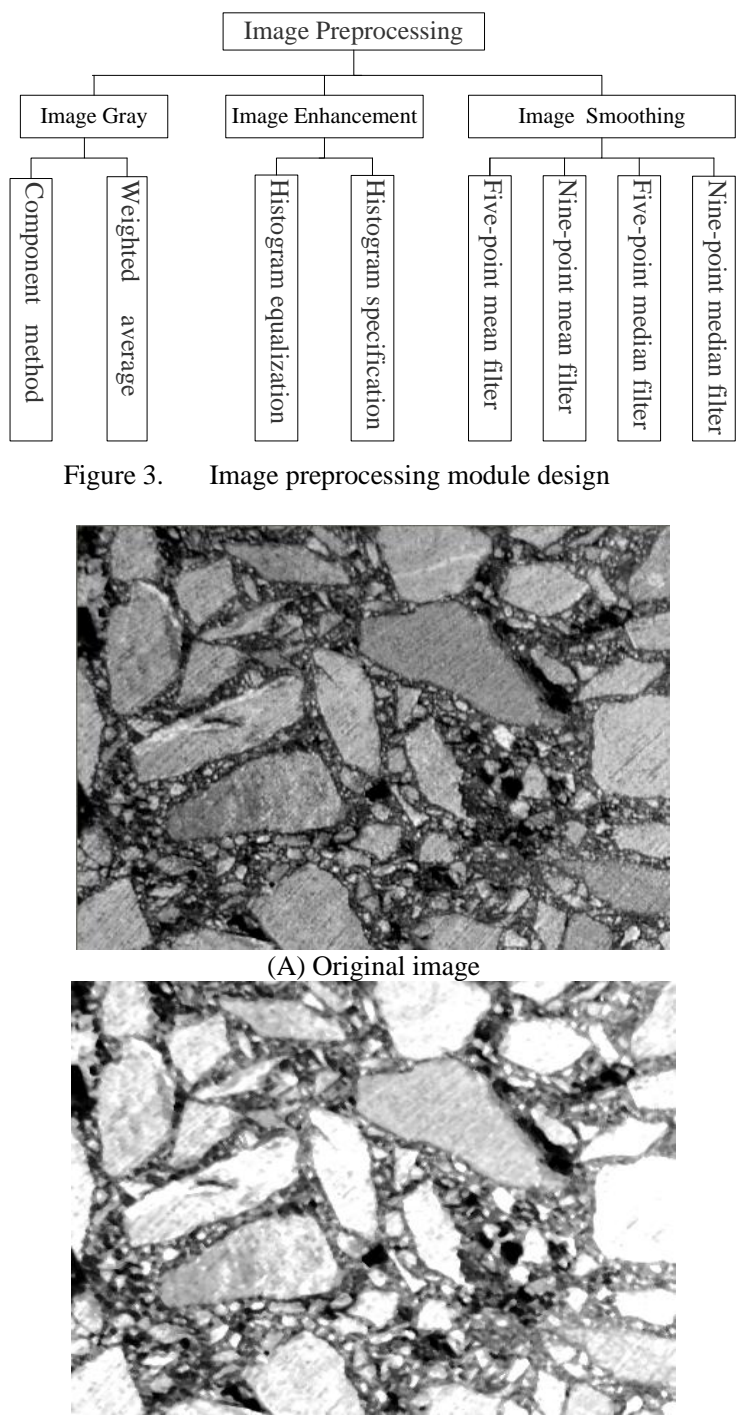

(B) Image after processing

Figure 4. Comparison of original and preprocessing image

Image Segmentation aims to separate the target object out of the background, so as to extract significant features of the object. The edge detection is achieved by gradient method, Roberts algorithm, sobel algorithm, Laplace algorithm etc.

Threshold selection is the key point in image binarization. This paper provides five ways to achieve the threshold selection. For different certain images, we can choose the most appropriate approach. Experiments show that the Otsu method can obtain satisfied results for all images. Mathematical morphology consists primarily of image erosion, dilation, opening operation and closing operation. The system realized all these four operations, so we can select different combinations to process asphalt mixture image depending on the certain situation in order to achieve the best results. The image segmentation module design is shown in Fig. 5 and the segmented image is shown in Fig. 6. 


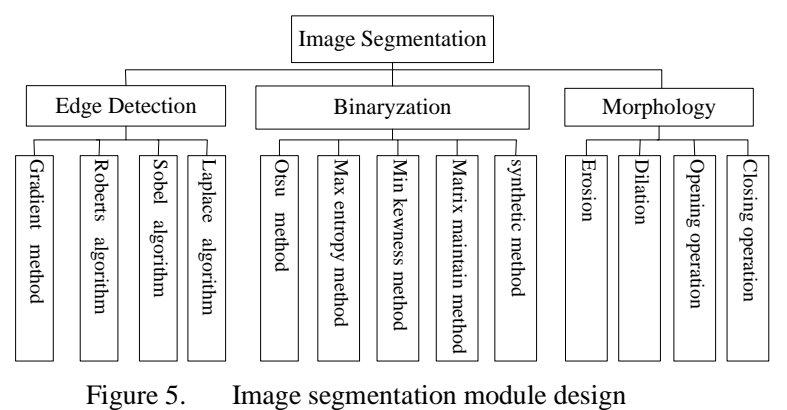

Figure 5. Image segmentation module design

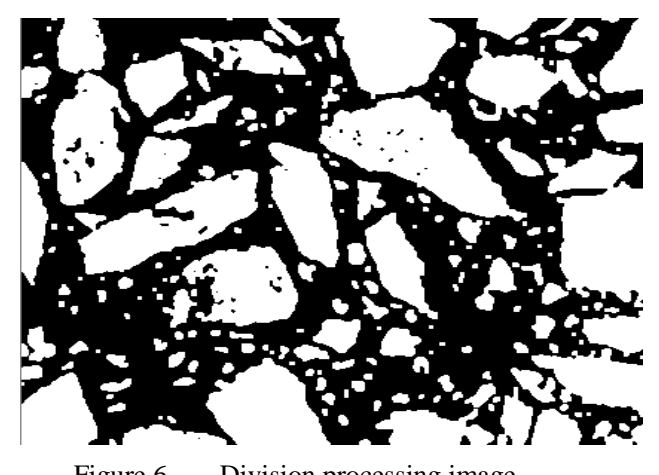

The main purpose of this paper is to obtain the characteristic parameters of the aggregate particles in the asphalt mixture image, including the geometric characteristics such as area, perimeter, shape factor, elongation, etc. In order to distinguish different particles, we labeled those segmented particles before parameter extraction, and show a message box with the maximum number, i.e. the total number of particles; besides, drawing particles' contour with red line. The result is shown in Fig. 7. The region-labeling algorithm is improved and it will be described in detail.

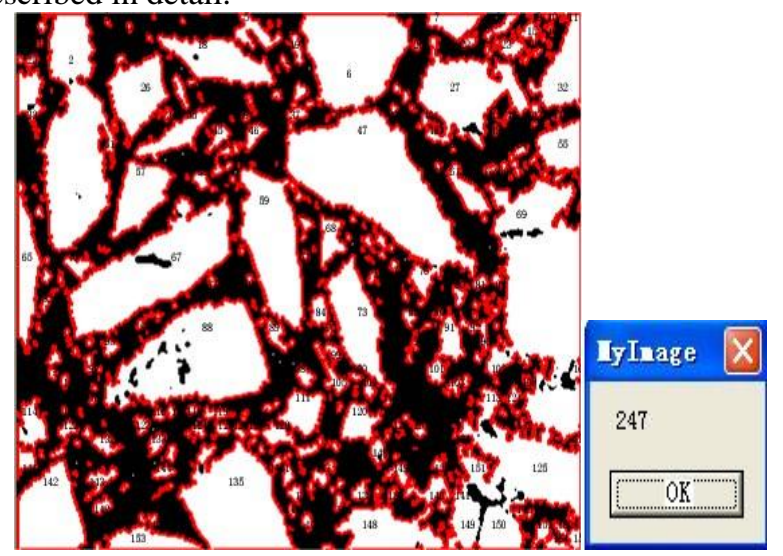

Figure 7. Region-labeling and contour drawing

After the above preprocessing, binarization and segmentation of the original asphalt mixture image, the target and background region has been separated, resulting in a corresponding binary image. Region-labeling algorithm is adopted in order to achieve recognition of different particles in the binary image and prepare for further characteristic parameter extraction of the mixed particles.
Binary image consists of the gray-scale pixels with value 0 (background) and with value 1 (particle).Region-labeling is endowing pixels of gray value 1 adjacent to each other with the same number, and different number to nonadjacent ones, thus achieve the distinction of different particles in the same image. Traditional connected component labeling method includes twice scanning of the binary image. First, do progressive scanning to the image and judge the neighborhood relationship of pixels, endow pixels belonging to the same regional connectivity with same number. Typical conflicts often occur that same pixel is repeatedly marked. As a consequence, the second scanning is needed which aims at merging sub-regions belonging to one connectivity into one region with the same label. The efficiency of this method is very low, especially in the case of high incidence of repetitive marking, the time complexity is greater.

Inspired by region-growth algorithm, a new method was introduced which marks one connected region one time and then marks the next area until all the connected regions in the image are marked instead of scanning the binary image by row or by column. Its computational overhead is mainly concentrated in two operations: one is scanning the binary image and searching for the starting point of the unlabeled region and the other one is 8-neighborhood searching of each pixel in the connected region. In the worst case, the algorithm needs to do eight neighborhood searches of all pixels in the input image. Assumed that the image has $n$ pixels, and the complexity of the algorithm at this situation is $\mathrm{O}(\mathrm{n})$ which is still much better than the traditional method. Besides, based on regional growth of eight neighborhood searches, the method characterized with good robustness which is not affected by area shape.

However, the algorithm treats new found qualified points as seed points of regional growth, which needs storing and taking out seeds constantly during the process and this will increase the whole system overhead and make the algorithm more complex. To solve this problem, further improvement on this algorithm has been done in this paper. Assume that the binary image $\mathrm{I}$ has $\mathrm{m}$ row and $\mathrm{n}$ columns, then the row $\mathrm{i}$, column $\mathrm{j}$ pixel is $\mathrm{P}(\mathrm{i}, \mathrm{j})$ and is endowed with $\mathrm{La}$. The improved labeling algorithm process is as follows:

(1) Progressive scanning the input image I with the purpose of finding the first point $\mathrm{P}(\mathrm{i}, \mathrm{j}$ ) belonging to an unlabeled region and mark the point with La;

(2) Check eight neighborhood points of $\mathrm{La}$, and determine whether it satisfies the connectivity requirements: (1) the gray-scale value is 1; (2)having not been tagged. Endowing the numeral $\mathrm{La}$ to the point that satisfies these two conditions, and then check the eight neighborhood points of the new labeled point, until the eight neighborhood pixels no longer satisfy the conditions(1)or (2). Thus one connected region, i.e. the marking of a particle is completed;

(3) Next, continue scanning to find the next point of unlabeled region and endow the pixel of a new particle with $\mathrm{La}=\mathrm{La}+1$. Repeating the process above until the entire asphalt mixture image is dealed with. So far, region-labeling process of an image is completed. The flowchart of the algorithm is shown in Fig. 8: 


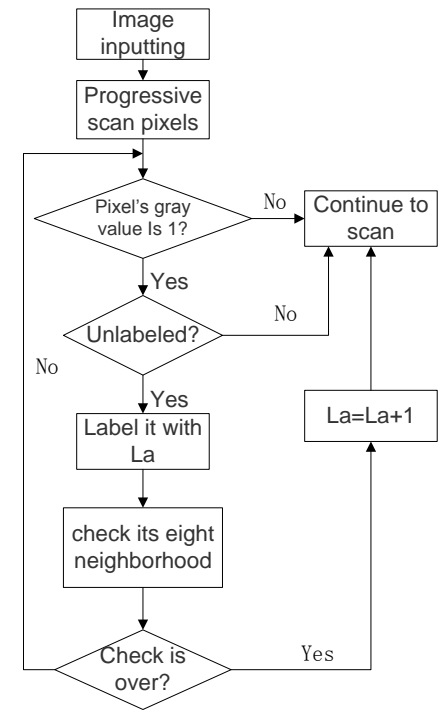

Figure 8. A modified particle labeling algorithm flowchart

Characteristic parameters of aggregate particles extracted from the image include the area, perimeter, shape factor, as well as elongation and other geometric characteristics.

The area of the target can be simply defined as the number of pixels that make up the object boundary. It is decided by the size of the target, regardless of its pixel gray values. After region-labeling process and contouring, the area and perimeter of the particle can be easily calculated using statistical methods. The calculation formula is as follows:

$$
A[n]=\sum_{i=0}^{n \text { Height }-1} \sum_{j=0}^{n \text { Width }-1} f(i, j) \quad n=0,1, \ldots, \text { num }-1
$$

Here num represents the label of the target regions in the image. A is a one-dimensional array which stores each target area. nHeight And nWidth denote the height and width of the processed image respectively. $f(i, j)$ represents the pixel gray value of the image where the pixel with $f(i, j)=1$ means particles pixel and $f(i, j)=0$ is not a particle pixel.

Progressive scan the binary image, if the point's gray value is 1 then plus 1 to its corresponding particle, otherwise continue to scan the next point. In this way, when the scanning is completed, areas of all particles in the image are obtained at the same time.

The perimeter of the area is the length region's boundary, which is calculated by a contour tracing method. Contour tracking aggregate particles in the image and take the number of boundary pixels as the corresponding particles' perimeter.

When boundary of the object is known, the easiest way to characterize its basic shape is to characterize it with the size of its bounding rectangle. To obtain a circumscribed rectangle of the object in the direction of the coordinate system, find out the maximum and minimum values of object in cross-ordinate direction respectively, then the length and width of the circumscribed rectangular can be obtained by formula (2), which can be used to represent the height and width of the aggregate particles approximately .

$$
b 2[k]=|\operatorname{Maxj}[k]-\operatorname{Minj}[k]|+1
$$

Here Maxi[k] ,Mini[k], Maxj[k], Minj[k] denote the maximum and minimum values of the ordinate as well as the maximum and minimum value of the abscissa of the $\mathrm{k}$-th particles respectively.

Circularity used to characterize the complexity of the object boundary. There are four kinds of circularity measure, including the density $\mathrm{Ci}$, boundary energy $\mathrm{E}$, the circular as well as the ratio of area and the average of the square of the distance. The circularity of particle labeled with $\mathrm{k}$ is calculated using the following formula:

$$
\mathrm{Ci}[\mathrm{K}]=\mathrm{Pe}[\mathrm{k}] 2 / \mathrm{A}[\mathrm{k}]
$$

That is the ratio between the square of the perimeter and the area. For roundness the value of the characteristics is $4 \pi$ which is the minimum. The more complex the shape is, the greater the value will be. The circularity index $\mathrm{Ci}$ has a rough contact with the boundary's complexity. The close degree between the target shape and the circle can be described with the shape factor: the more closer its shape is to a circle, the greater is the value. The formula is as follows, where A [k] denotes the area of the k-th particle, Pe [K] represents the perimeter of the $\mathrm{k}$-th particle.

$$
\mathrm{C}[\mathrm{k}]=4 \pi * \mathrm{~A}[\mathrm{k}] / \mathrm{Pe}[\mathrm{k}] 2
$$

The rectangularity reflects the full extent of the circumscribed rectangle of the object, usually described with the ratio of object's area and the area of its smallest circumscribed rectangular.

$$
\operatorname{Re}[\mathrm{k}]=\mathrm{A}[\mathrm{k}] /(\mathrm{b} 1[\mathrm{k}] * \mathrm{~b} 2[\mathrm{k}])
$$

Where A [k]represents the area of the K-th particles, b1 $[\mathrm{k}]$, b2 $[\mathrm{k}]$ denote the length and width of the minimum bounding rectangle of the particles respectively. The value range of $\operatorname{Re}$ is between 0 and 1 , and $\operatorname{Re}$ reaches the maximum 1.0 when the object is a rectangle while the $\mathrm{Re}$ value of circular object is $-\pi / 4$. The Re value of slender, curved object is smaller.

Elongation is another parameter reflects the shape of the particle, and the formula is shown as follows:

$$
\mathrm{T}[\mathrm{k}]=(\mathrm{b} 1[\mathrm{k}] * \mathrm{~b} 2[\mathrm{k}]) / \mathrm{A}[\mathrm{k}]
$$

A [k] denotes the area of the k-th particles, b1 [k], b2 [k] denote the length and width of the minimum bounding rectangle of the particles respectively.

The database module deals with the storage, query and print of the results. Build a database in SQL Server 2000, the processing result of every image handled by the system is written to a specific table. Click the corresponding button 
in the main interface, and you can view the data in the database. Users can look up the parameters of specific particle indexed by ID number, area range and so on; besides results can be printed if needed.

Parts of the characteristic parameter calculating results of particles are shown in Fig. 9.

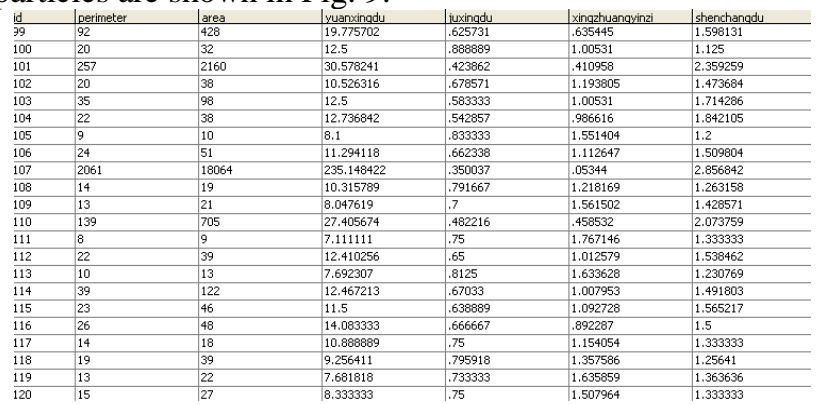

Figure 9. Particles characteristic parameter calculating result

\section{CONCLUSIONS}

This article is based on Visual Studio 2008 and SQL Server 2000 achieving the characteristic parameter extraction of the asphalt mixture aggregate and processing the storage and query of the results. Some basic techniques of digital image processing are adopted, including image preprocessing, image enhancement, edge detection, binarization, morphology processing, as well as region-labeling and contouring. Besides, the region-labeling algorithm has been improved, which improved processing efficiency greatly. Finally, the database was designed and realized the storage, query and print of those results. Practice has proved that the system has a high value in use and a broad application prospects. Although this system has provided an automated method of characteristic parameter extraction o f aggregate particles with very high practical value, further study still needs to be done in the future. For example, the whole system especially the database could be designed based on distribution technology, so more users with authorization could get access to it more easily and conveniently. Processing object is single image in this paper, a new system can be developed to achieve sequential processing of images.

\section{ACKNOWLEDGMENT}

This work is supported by Specialized Research Fund for the Doctoral Program of Higher Education (Grant No. 20110205110005), which is gratefully appreciated.

Fund Project: Specialized Research Fund for the Doctoral Program of Higher Education (Grant No.20110205110005)

\section{REFERENCES}

[1] D.Penumadu, Strain Localization in Solid Cylindrical Clay Specimens Using Digital Image Analysis Technique [J]. Soils and Foundations, 2007.Vol.47 (1),PP. 67-78.

[2] Xiao-ning Zhang. Designing asphalt mixture [J]. Harbin University of C. E. \& Architecture. 2002. Vol.35 (1), PP. 108-111

[3] Hui-ling Zhang, Zhao-yun Sun. Study on Asphalt Mixture Automatic Measurement and Interface Technology [J]. onstruction Machinery \& Construction Technology, 2009.Vol.26 (4), PP. 42-45
[4] Zhao-yun Sun, Ai-min Sha, Qiu-Ling Yao. Research on the Automatic Measurement System of the Asphalt Mixture [J].Chinese Journal of Scientific Instrument.2006.Vol.27 (4), PP. 353-357

[5] Yu-jin Zhang. Image Engineering (Volume one) image processing (2nd edition) [M]. Beijing: Tsinghua University Press, 2006.

[6] Jianfang Shi, Fujun Zhang, Baofeng Hao. Image segmentation algorithms based on wavelet transform and mathematical morphology[J]. Technology journal of Taiyuan University. 2009.Vol.40 (5), PP. 490-493

[7] Wenjun Tong, Jun Zhang. Target detection algorithm based on edge detection and the traditional difference method[J].2011.Vol.21 (2):37-40

[8] Hongjiu Tao, Jian Liu,Jinwen Tian. Edge detection for remote sensing image based on wavelet transform and mathematical morphology[J]. Infrared and laser engineering. 2002.Vol.31 (2), PP. $154-157$

[9] Zhicheng Dai. actual combat training on Chinese version of SQL Server database programming[M]. Beijing: People's Posts and Telecommunications Press, 2004.

[10] Bosheng Chen. A binary image connected component labeling method [J], Computer Engineering and Application, 2006 